\title{
Hidroxiapatita de cálcio: uma revisão quanto à eficacia, segurança e imaginologia quando usado como preenchedor e como bioestimulador
}

\author{
Calcium hydroxyapatite: a review for efficacy, safety and imaging when used as a filler and as a \\ biostimulator
}

Hidroxiapatita de calcio: una revisión de la eficacia, seguridad e imagenología cuando se usa como relleno y como bioestimulador

Recebido: 12/10/2021 | Revisado: 19/10/2021 | Aceito: 20/10/2021 | Publicado: 23/10/2021

\author{
Cristiani Sandrelli Ferreira Pacheco de Oliveira \\ ORCID: https://orcid.org/0000-0002-7143-408X \\ Associação Brasileira de Odontologia, Brasil \\ E-mail: dracristiani@gmail.com \\ Tereza Jacy da Silva Almeida \\ ORCID: https://orcid.org/0000-0003-1059-1978 \\ Associação Brasileira de Odontologia, Brasil \\ E-mail: terezaja@gmail.com \\ Luciene de Oliveira Martins \\ ORCID: https://orcid.org/0000-0001-5984-2067 \\ Associação Brasileira de Odontologia, Brasil \\ E-mail: luciene.o.martins@gmail.com \\ Lara Andréa Torelli Marques Sorpreso \\ ORCID: https://orcid.org/0000-0001-7227-1086 \\ Associação Brasileira de Odontologia, Brasil \\ E-mail: larasorpreso@gmail.com \\ Nathalia Silveira Finck \\ ORCID: https://orcid.org/0000-0002-8435-2390 \\ Rede de Ensino Doctum, Brasil \\ E-mail: nathaliafinck@gmail.com
}

\begin{abstract}
Resumo
Este artigo teve como objetivo apresentar por meio de uma revisão de literatura a eficácia, segurança e satisfação dos pacientes com o uso de hidroxiapatita de cálcio como preenchedor e como bioestimulador de colágeno. Trata-se de uma revisão de literatura realizada por meio de busca ativa dos artigos cientificos na base de dados: U.S. National Library of Medicine (PubMed), utilizando como termo de busca: calcium hydroxylapatite cosmetic. Foram selecionados 69 publicações, no periodo de 2016 à 2021, entretanto à partir dos critérios de inclusão e exclusão foram selecionados 20 artigos. Baseado na revisão de literatura feita neste estudo, a hidroxiapatita de cálcio mostrou-se eficaz como bioestimulador de colágeno e elastina; como preenchedor apresentou um melhor efeito lift (exceto no caso do lift de sobrancelha) por causa do seu alto módulo de elasticidade, podendo ser usado como suporte para outros preenchedores e fios de sustentação; teve um elevado nivel de satisfação dos pacientes; é considerado um material com um bom perfil de segurança, desde que respeitada a técnica e as indicações; e pode ser visualizado em radiografias, tomografias e ultrassonografias de alta resolução.
\end{abstract}

Palavras-chave: Hidroxiapatita de cálcio; Estética; Preenchedores dérmicos.

\begin{abstract}
The purpose of this article was to present, through a literature review, the effectiveness, safety and satisfaction of patients with the use of calcium hydroxyapatite as a filler and as a collagen biostimulator. This is a literature review carried out through an active search for scientific articles in the database: U.S. National Library of Medicine (PubMed), using the search term: calcium hydroxylapatite cosmetic. Sixty nine publications were selected, from 2016 to 2021, however, based on the inclusion and exclusion criteria, 20 articles were selected. Based on the literature review carried out in this study, calcium hydroxyapatite was effective as a biostimulator of collagen and elastin; as a filler it presented a better lift effect (except in the case of eyebrow lift) because of its high modulus of elasticity, which can be used as a support for other fillers and support threads; had a high level of patient satisfaction; it is considered a material with a good safety profile, as long as the technique and indications are respected; and can be viewed on radiographs, tomographies and high resolution ultrasounds.
\end{abstract}

Keywords: Calcium hydroxylapatite; Esthetics; Dermal fillers. 


\begin{abstract}
Resumen
Este artículo tuvo como objetivo presentar, a través de una revisión de la literatura, la eficacia, seguridad y satisfacción de los pacientes con el uso de hidroxiapatita cálcica como relleno de colágeno y como bioestimulador de colágeno. Se trata de una revisión de la literatura realizada a través de una búsqueda activa de artículos científicos en la base de datos: Biblioteca Nacional de Medicina de EE. UU. (PubMed), utilizando como término de búsqueda: cosmético de hidroxiapatita cálcica. Se seleccionaron 69 publicaciones de 2016 a 2021, sin embargo, en base a los criterios de inclusión y exclusión, se seleccionaron 20 artículos. Con base en la revisión de la literatura realizada en este estudio, la hidroxiapatita de calcio demostró ser eficaz como bioestimulador de colágeno y elastina; como relleno presentó un mejor efecto lifting (excepto en el caso del lifting de cejas) por su alto módulo elástico, que puede ser utilizado como soporte para otros rellenos y alambres de soporte; tuvo un alto nivel de satisfacción del paciente; se considera un material con un buen perfil de seguridad, siempre que se respete la técnica y las indicaciones; y se puede visualizar en radiografías de alta resolución, TC y ecografías.
\end{abstract}

Palabras clave: Hidroxiapatita de cálcio; Estética; Rellenos dérmicos.

\title{
1. Introdução
}

O aumento da longevidade fez com que as pessoas buscassem cada vez mais tratamentos estéticos e funcionais, com a intenção de gerenciar o envelhecimento e objetivando resultados mais naturais e harmoniosos. Nos últimos anos, além da busca deste rejuvenescimento natural e harmonioso, muitas pessoas buscam o embelezamento no sentido de "corrigir" características faciais que não lhes são agradáveis. Para que isto aconteça, se faz necessário intervir em planos diferentes da face, uma vez que o envelhecimento é o resultado de mudanças nas cinco camadas anatômicas da face: ossos, ligamentos, músculos, tecido adiposo e pele (De Almeida, et al., 2019; Wollina, et al., 2020).

Do mesmo modo, tem-se observado o aumento na busca de procedimentos não cirurgicos para tratamento de rejuvenescimento do rosto e corpo por causa do reduzido tempo de inatividade e menor chance de complicações (Guida, et al., 2020).

A hidroxiapatita de cálcio é um dos materiais que está sendo utilizado para atingir este objetivo. Ele foi inicialmente aprovado pela US Food and Drug Administration (FDA), em 2001, como um implante injetável para ser um marcador radiográfico em tecido mole. Mas em 2006, a FDA aprovou Radiesse® (Merz Aesthetics, Raleigh, NC) como um preenchedor à base de hidroxiapatita de cálcio para uso em sulcos naso-labiais e em pacientes com vírus HIV associados com lipoatrofia facial (Goldberg, et al., 2018).

Radiesse ${ }^{\circledR}$ consiste em osso sintético não imunogênico, composto em $30 \%$ por microesferas de hidroxiapatita de cálcio de 25 a 45 um de diâmetro, que encontram-se mergulhadas na abundante matriz, equivalente a $70 \%$ do produto, do gel de carboxi-metilcelulose de sódio(Goldberg, et al., 2018; Lee \& Lorenc, 2016; Courderot-Masuyer, et al., 2016; Wollina, et al., 2020; Loghem, et al., 2015).

A Hidroxiapatita de Cálcio é considerada um material preenchedor semi-permanente e quando diluído é essencialmente um bioestimulador de colágeno (Goldberg, et al., 2018; Lorenc, et al., 2018).

Semanas após o material ser injetado, o gel de carboximetilcelulose é absorvido e um volume neutro líquido aparece através da neocolagênese. A hidroxiapatita de cálcio se degrada em íons cálcio e fosfato, sendo excretado lentamente pelo corpo, propiciando um efeito de volume duradouro por volta de 12 a 18 meses (Loghem, et al., 2015; Goldberg, et al., 2018).

Com a mudança de mentalidade no gerenciamento do envelhecimento, no sentido de tratar de forma tridimensional com restituição de volume, ao invés de preencher rugas isoladas, e com o aumento de preenchedores disponíveis no mercado; se faz necessário entender as características fisicoquímicas e conhecer as propriedades de cada preenchedor, associando ao seu desempenho, imunogenicidade, aplicações, resultados estéticos, entregabilidade e longevidade para que o profissional tenha como decidir qual material será melhor aplicado em cada situação clínica (Liu, et al., 2018). 
Este artigo teve como objetivo apresentar por meio de uma revisão de literatura a eficácia, segurança e satisfação dos pacientes com o uso de hidroxiapatita de cálcio como preenchedor e como bioestimulador de colágeno.

\section{Metodologia}

Trata-se de uma revisão de literatura, cujo levantamento bibliográfico foi realizado na seguinte base de dados eletrônica: U.S. National Library of Medicine (PubMed). Para isso, foi utilizado como termo de busca: calcium hydroxylapatite cosmetic, e foram encontradas 69 publicações no corte transversal entre os anos de 2016 a 2021 e selecionados artigos na língua inglesa. Os artigos mais antigos foram descartados, deixando apenas um de 2009 por sua relevância. O critério de inclusão dos artigos utilizados no presente estudo se deu independente de relatos dos estudos em corpo ou face, considerando temas como eficácia do tratamento, segurança e imagenologia. Quanto à eficácia, consideramos dados histológicos, pesquisa in vitro mas também a satisfação do paciente. Foram selecionados 20 artigos seguindo os critérios de inclusão previamente citados.

\section{Fundamentação Teórica}

\subsection{Bioestimulador de colágeno}

A hidroxiapatita de cálcio (CaHA) tem sido usada como bioestimulador de colágeno, ou seja, é um material biocompatível e indicado para estimular a produção de colágeno. Com o objetivo de examinar o efeito da CaHA na produção de colágeno, Zerbinati \& Calligaro (2018) avaliaram tecidos que receberam injeção subdérmica de 0,3ml de CaHA (Radiesse $\AA_{\text {), }} 2$ meses antes de serem removidos durante cirurgia plástica abdominal, quando também foram removidos tecidos das mesmas pacientes numa distância de $10 \mathrm{~cm}$ para serem utilizados como grupo controle. $\mathrm{O} \mathrm{n}$ desta pesquisa foi 5 , os tecidos receberam um corante histoquímico específico para colágeno (picrosirius red $0,1 \%$ ) e foram avaliados em microscopia de luz polarizada circular (Zeiss Axioplan). Nos sítios que receberam CaHA, Zerbinati encontrou uma proporção relativamente maior de fibras que representam o colágeno tipo III (colágeno novo) e no grupo controle mostrou composição integralmente de colágeno tipo I (colágeno maduro). Normalmente existe uma proporção entre colágeno tipo I e tipo III e esta proporção depende de fatores como a idade do indivíduo. E durante a neocolagênese ocorre uma maturação do colágeno tipo III em colágeno tipo I que apresenta uma resistência à tração e suporte estrutural ideal. O autor complementa que como as biopsias foram feitas após 2 meses da aplicação da CaHA, talvez tenha sido cedo para detectar o pico máximo da produção de colágeno, mas seu estudo confirma que CaHA estimula formação de colágeno tipo III.

A hidroxiapatita de cálcio é utilizada em tratamentos faciais, mas também em tratamentos corporais. Casabona et al. (2017) pesquisaram o uso da hidroxiapatita de cálcio diluída 1:1 associada com microagulhamento e ácido ascorbico tópico $20 \%$ em estrias brancas e vermelhas. Nesta pesquisa o n foi de 35, sendo 25 de estrias vermelhas e 10 de estrias brancas. O tratamento consistiu de 3 sessões, sendo que na primeira foi feita a aplicação de CaHA diluída 1:1 seguida de vigorosa massagem e em seguida foi feito microagulhamento (agulha de $2 \mathrm{~mm}$ ) com Dermapen (Dermapen Fractional Micro Needle System; DermapenWorld Fort Lauderdale, Fla.) e aplicação tópica de ácido ascórbico 20\%. Na segunda sessão (1 mês após a primeira) e na terceira sessão ( 2 meses após a primeira) foi feito apenas o microagulhamento e aplicação tópica do ácido ascórbico. A avaliação foi feita 1 mês após a última sessão do tratamento. Uma das pacientes que foi submetida ao tratamento ia fazer uma abdominoplastia e concordou em fazer biopsia de tecido para análise histológica. Com o intuito de comparação, ela recebeu o tratamento proposto numa área e em outra área foi feito apenas microagulhamento e ácido ascórbico tópico. Foram removidas 3 amostras: uma da área tratada com CaHA, microagulhamento e ácido ascórbico tópico, outra da área onde foi feita apenas microagulhamento e ácido ascórbico tópico e outra de uma área que não recebeu tratamento. Histologicamente, 
observou-se que a área que recebeu CaHA, microagulhamento e ácido ascórbico tópico teve uma melhora na qualidade e na quantidade de colágeno e fibras elásticas na derme, quando comparada com a área que foi submetida apenas ao microagulhamento e ácido ascórbico tópico e à área que não recebeu tratamento. Nas papilas dérmicas (subepiderme) também se observou aumento da quantidade de colágeno na área que recebeu microagulhamento e ácido ascórbico tópico quando comparada com a área não tratada. Com estes resultados, a autora concluiu que esta associação pode produzir aumento de colágeno na pele.

Guida, et al., (2020) estudaram o uso da hidroxiapatita diluida de 1:4 em 20 joelhos (de 20 mulheres), no tratamento de flacidez de pele e celulite na regiao acima dos joelhos. Os resultados demonstraram uma melhora significativa na aparencia estetica da região estudada.

Em 2021, Guida, et al., avaliaram a eficácia e segurança do uso de hidroxiapatita diluida de 1:2 em 20 pescoços, no tratamento de flacidez de pele. Foram feitas fotografias antes do procedimento e após 3 meses. A classificação foi feita segundo a escala NSL (neck skin laxity) e o teste T-student foi aplicado. Este estudo suportou a eficácia e a segurança do tratamento feito com hidroxiapatita diluida 1:2 para flacidez da pele de pescoço, segundo a escala NSL.

Fabi, et al., (2017) relataram em sua publicação, um sumário das apresentações feitas num meeting que aconteceu em Praga em novembro de 2016 e reuniu mais de 400 profissionais da medicina estética, dermatologia e cirurgia plástica. O objetivo deste encontro foi compartilhar com os profissionais sobre os avanços recentes da medicina estética com o foco em intervenções combinadas para a prevenção, rejuvenescimento da pele envelhecida, aspectos multiculturais e expectativa dos pacientes. Entre as intervenções associadas, o uso da hidroxiapatita de cálcio foi considerado um efetivo tratamento para melhorar contorno e firmeza em braços superiores, além do uso em face, pescoço, colo e mãos. Concluíram dizendo que a hidroxiapatita de cálcio diluída melhora as propriedades mecânicas da pele.

Ainda considerando a eficácia, Courderot-Masuyer, et al., (2016), em seu estudo, tiveram como objetivo ver a diferença entre a força contrátil dos fibroblastos naturais e envelhecidos e dos fibroblastos de sulcos e rugas, na presença ou não da hidroxiapatita de cálcio, e para isto propôs uma pesquisa in vitro. Ele percebeu que na ausência do bioestimulador, a força contrátil dos fibroblastos naturais, porém envelhecidos é significativamente maior que os fibroblastos presentes em rugas e sulcos. Quando utilizada a hidroxiapatita de cálcio, os fibroblastos naturais e envelhecidos que sofreram a ação do bioestimulador apresentaram força contrátil maior que na ausência deles. O mesmo acontecendo com os fibroblastos presentes em rugas e sulcos que também apresentaram uma força contrátil maior quando submetidos aos efeitos da hidroxiapatita de cálcio. Desta forma, ele concluiu dizendo que seu estudo mostrou um efeito positivo da hidroxiapatita de cálcio no envelhecimento pois observou-se que na presença deste estimulador houve um aumento das forças de contração dos fibroblastos presente em rugas e sulcos.

Em 2019, De Almeida, et al., propuseram um estudo feito a partir de um questionário entregue e respondido por 10 especialistas que atuam nas áreas de dermatologia e cirurgia plástica, que estavam reunidos num Meeting que aconteceu em São Paulo (Brasil) em novembro de 2017. O objetivo deste estudo foi desenvolver Recomendações que fossem consenso entre a maioria deles quanto à segurança e eficácia da CaHA hiperdiluida, ou seja, como bioestimulador. Este estudo concluiu que Radiesse (CaHA) é associada a um alto perfil de segurança e efetividade para aumento da qualidade e firmeza da pele, sendo um produto recomendado para esta busca cada vez mais crescente de soluções rejuvenescedoras e minimamente invasivas.

\subsection{Preenchedor}

Lee e Lorenc (2016), em seu artigo, relataram que o Radiesse (hidroxiapatita de cálcio) tem um alto modulo de elasticidade. Considerando que módulo de elasticidade é a habilidade que um material tem de resistir deformação quando uma pressão é aplicada sobre ele, quanto maior o modulo de elasticidade, maior sua resistência, e menor sua capacidade de 
deformar quando submetido à pressão. Esta propriedade confere à hidroxiapatita de cálcio um melhor efeito lift quando injetado sob a pele como preenchedor.

Além do modulo de elasticidade, Lorenc, et al., (2018) também estudaram outras características físico-quimicas do preenchedor CaHA, como a sua viscosidade, sua reologia e o seu efeito bioestimulador. Baseado em seus estudos, ele declara que em seu perfil reológico, a CaHA apresenta um modulo de elasticidade e viscosidade maior que o preenchedor à base de acido hialurônico. Esta conclusão oferece bases científicas para considerar a CaHA um preenchedor que serve como um implante líquido e um excepcional tecido de suporte para revolumização facial.

Da mesma forma, Goldberg, et al., (2018) reconheceram que a CaHA é uma valiosa ferramenta quando as características de um implante líquido são desejadas. Os autores concluem que o alto modulo de elasticidade da CaHA, the confere esta característica de implante líquido, especialmente em áreas de difícil tratamento como queixo e testa. Eles também concluem que considerando as abordagens em camadas para restauração de volume, o uso da CaHA contribuiu para resultados melhores; pois provê um suporte que serve de "andaime" para outros tratamentos complementares como outros preenchedores ou outras intervenções como os fios de sustentação, podendo levar a ótimos resultados.

Segundo Fakin-Gomez e Kaudoch, (2021) muitos medicamentos sao usados off-label e o uso do acido hialuronico (AH) e da hidroxiapatita de calcio (CaHA) na mesma sessão é um caso desses. Eles podem ser injetados separadamente ou misturados previamente à injeção. Quando usados no mesmo plano porem com imjeções diferentes, eles mantem suas propriedades reológicas. Quando misturados previamente e aplicados na mesma injeção, suas propriedades reológicas são alteradas e ainda não foram definidas. O presente estudo avaliou se o resultado foi mais eficaz e qual foi a duração do resultado obtido. Eles concluiram que a mistura do acido hialuronico e da hidroxiapatita de calcio foi eficaz para volumizar e dar um efeito lifting na mandibula e no queixo. Os resultados sugerem que a satisfação com 3 meses após o tratamento, foi maior com o uso da mistura de $\mathrm{AH}$ e da CaHA do que quando o AH foi usado sozinho. Com 12 meses, esta maior satisfação desaparece, talvez pelo efeito final do $\mathrm{AH}$.

\subsection{Satisfação}

Bass, et al., (2009) compararam o uso de hidroxiapatita de cálcio (Radiesse®) e de colágeno humano (Cosmoplast, Inamed, Santa Barbara, California) em sulcos nasolabiais. Foram analisados 117 pacientes com sulcos nasolabiais moderados a severos, onde utilizaram hidroxiapatita de cálcio de um lado $(1,2 \mathrm{ml})$ e colágeno humano $(2,4 \mathrm{ml})$ do outro lado, com critério para que não houvesse assimetria. Foram feitas fotos de cada lado e analisadas separadamente (duplo cego) em 3 e 6 meses. Seu objetivo era analisar segurança e eficácia num prazo de 3 anos. Os pacientes foram avaliados com 1, 3, 6 e 12 meses. Depois foram chamados com 24 e 36 meses para novas avaliações. Com 6 meses, o lado que havia recebido colágeno humano, foi tratado com CaHA para corrigir assimetria. Dos 117 pacientes, 102 voltaram com 24 meses, e destes 102, voltaram 99 com 36 meses. Os efeitos adversos apresentados foram eritema, edema e equimose na primeira semana após injeção, sendo mais frequente no lado tratado com CaHA do que do lado tratado com colágeno humano, possivelmente porque para a CaHA foi utilizada injeção com agulha de 27 gauge e o colágeno humano com agulha de 30 gauge. Observou-se 1 nódulo no lado tratado com CaHA e 3 nódulos no lado tratado com colágeno humano. Estes efeitos adversos foram resolvidos sem que houvesse necessidade de intervenção. E no retorno de 24 e 36 meses não se observou efeitos adversos. Quanto à eficácia, com 6 meses, 94,6\% dos pacientes que receberam CaHA estavam muito satisfeitos com o resultado contra 2,7\% dos que receberam o colágeno humano; e com 30 meses após a última injeção, a satisfação com o CaHA foi de 40\%. Concluindo assim, que a satisfação dos pacientes quanto ao resultado, foi maior com o uso da CaHA do que com o colágeno humano. 
No tratamento de estrias com o uso associado de CaHA, microagulhamento e ácido ascórbico 20\%, proposto por Casabona e Marchese (2017), conforme acima citado, ela também avaliou a eficácia do tratamento e observou que 85,8\% das pacientes que receberam tratamento ficaram satisfeitas ou muito satisfeitas com o resultado da terapia. A autora concluiu que este tratamento foi uma terapia satisfatória.

O estudo de Guida, et al., (2020) da hidroxiapatita de calcio diluida 1:4 e aplicada na regiao acima dos joelhos para tratamento de flacidez e celulite, sugere que o tratamento precoce seja mais eficaz do que quando realizado em mulheres com o grau de comprometimento mais severo. Os resultados demonstram que este tratamento é seguro e eficaz, com 100\% das pacientes satisfeitas.

Por outro lado, Liu, et al., (2018) relataram um caso clínico de uma paciente de 44 anos que foi submetida a tratamento com hidroxiapatita de cálcio (Radiesse@), com o objetivo de elevar a sobrancelha, mas neste caso a paciente não percebeu nenhum efeito lift na sobrancelha como desejado.

Wollina, et al., (2020) relatou um estudo com 40 mulheres entre 50 e 95 anos que receberam injeção de hidroxiapatita de cálcio (Radiesse $\left.{ }^{\circledR}\right)$ e que repetiram o procedimento com 12 a 14 meses em média e foram avaliadas até 6 anos após a primeira injeção. Mulheres que tinham feito lifting cirurgico e fios não foram incluidas no estudo. A avaliação de satisfação foi feita pelo proprio paciente e pelo profissional. O resultado foi bem parecido e sugeriu uma notavel melhora após o primeiro procedimento e a longo prazo demonstrou uma significativa e duradoura melhora da aparencia facial.

\subsection{Segurança}

Quanto à segurança, Casabona e Marchese (2017) observaram que no tratamento com CaHA, microagulhamento e ácido ascórbico tópico 20\% para estrias brancas e vermelhas, as pacientes apresentaram hematoma e eritema mas que foram resolvidos com 7 dias e também tiveram leve dor que desapareceu com 2 dias. Das 35 pacientes tratadas, 2 pacientes com fototipo III (Fitzpatrick) desenvolveram hiperpigmentação pós inflamatórias, sendo totalmente resolvido com o tratamento a que foram submetidas durante 30 dias com creme clareador (Kligman’s formula). Não observou nenhum evento adverso sério, concluindo assim que é uma terapia segura.

Da mesma forma, o estudo comparativo entre a hidroxiapatita de cálcio e o colágeno humano, proposto por Bass (1) demonstrou que a hidroxiapatita de cálcio é tão segura quanto o colágeno humano.

Kaudoch (2007) fez uma revisão de literatura com o objetivo de avaliar a segurança e as possíveis complicações com o uso da hidroxiapatita de cálcio. Vinte e um artigos publicados entre 2004 e 2015 foram incluídos, totalizando 5081 tratamentos com CaHA em 2779 pacientes. Dos 5081, ele encontrou 173 eventos adversos que representam 3\% do total dos tratamentos realizados. Destes 173 eventos adversos, a maioria foram nódulos (96\%) e principalmente em lábios. A maioria foi tratada com massagem e corticoide intralesional e apenas 10 casos dos 166 (6\%) precisou fazer excisão do nódulo. Baseado neste estudo, o autor conclui dizendo que a CaHA aparece como um material que tem um bom perfil de segurança, e ressaltou que se ele não for utilizado em áreas dinâmicas da face, como lábios e região peri-orbital, haverá menor possibilidade de incidência de nódulos.

Loghem, et al., (2015) descreveram uma revisão de literatura para marcar os 10 anos de uso da hidroxiapatita de cálcio no campo da estética. Eles forneceram um protocolo detalhado do seu uso, assim como consideraram as propriedades únicas da mesma, o que faz com que ela ocupe um lugar importante no mercado da estética atual. Eles concluem que é um material muito eficaz e que apresenta um perfil de segurança alto e bem estabelecido. Eles relatam que pode ocorer a formação de nodulos quando a CaHA é injetada nos labios e na mucosa oral, sendo que esta indicação não é aprovada pelo fabricante. 
Na declaração de consenso geral sobre o uso da hidroxiapatita de cálcio, De Almeida, et al., (2019) citam o nódulo como possível evento adverso e alertam que quando se deseja volume, embora algumas preocupações como necrose tecidual e complicações serias como embolismo sejam citadas na literatura, nenhum comprometimento vascular foi observado pelos autores em sua prática clínica e nem por Kaudoch (2007) em sua revisão de literatura. Entretanto, cautela sempre será a regra quando se tratar de face; e áreas de alto risco como área glabelar e nariz devem ser evitadas.

Da mesma forma, Lee e Lorenc (2016) relatam que as raras complicações de uso de hidroxiapatita de cálcio incluem nódulos e oclusão vascular. Apesar de não existir agentes de reversão ou enzima para hidroxiapatita de cálcio, nódulos podem ser quebrados com massagem manual. No caso de nódulos maiores, a recomendação é que sejam tratados com injeções intralesionais de 5-fluorouracil e lidocaína 1:1 para redução da ação dos fibroblastos naquele local. Nos casos de oclusão vascular, que são extremamente raros, recomenda-se o mesmo protocolo utilizado para os outros preenchedores, incluindo o uso de hialuronidase.

Apesar de não existir um agente de reversão para a hidroxiapatita de calcio, assim como existe a hialuronidase para o ácido hialurônico; o uso off-label do tiossulfeto de sódio tem sido sugerido. Rullan, et al., (2019) relataram dois casos clinicos de nódulos visíveis de hidroxiapatita de cálcio, cujo tratamento com o tiossulfeto de sódio foi bem-sucedido.

Danyz, et al., (2020) por sua vez, fez um estudo pré-clinico com o tiossulfeto de sódio usando diversos métodos de verificação, tais como camera 3D, tomografia micro-computadorizada ex vivo, tomografia computadorizada in vivo, histopatologia, microscopia eletronica scanner. Eles concluiram que apesar do tiossulfeto de sodio ser mais eficaz que a solução salina no tratamento de nódulos de hidroxiapatita de calcio, a solução salina apresentou maior segurança uma vez que o tiossulfeto de sodio apresentou risco de necrose tecidual e hemorragia. Além disso, o uso de tiossulfeto de sódio nao resolveria os casos de oclusão vascular uma vez que seu mecanismo de ação sugerido foi de dispersão e não de degradação.

Loghem, et al., (2020) descreveram que apesar de ser uma raridade, injeção intra-vascular de hidroxiapatita de cálcio pode acontecer, assim como pode acontecer com qualquer preenchedor. Estas injeções podem não trazer sequelas mas em alguns casos podem causar necrose tecidual e cegueira, sendo mais comum quando utilizado no dorso do nariz. Sendo assim, todo profissional deve estar preparado para identificar os sinais e sintomas precocemente e intervir o mais rápido possível.

No estudo de Wollina, et al., (2020) observou-se 6 eventos adversos, sendo 4 hematomas e 2 nódulos não visíveis, os quais foram resolvidos sem intervenção e num periodo de 2 a 4 semanas.

Guida, et al., (2020) concluiram no seu estudo que o uso da CaHA diluida 1:4 na regiao acima dos joelhos é segura pois os únicos eventos adversos foram eritema, edema e equimose que foram resolvidos espontaneamente e pele irregular que durou pouco tempo após uma massagem vigorosa. E em 2021, Guida, et al., concluíram que o uso da hidroxiapatita diluída 1:2 foi segura para tratamento de flacidez de pele no pescoço, pois os efeitos adversos apresentados foram vermelhidão, edema e hematoma que foram resolvidos entre 2 e 14 dias, sem apresentar nenhuma sequela.

Lee e Lorenc (2016) também dizem em sua publicação, que sendo a hidroxiapatita um osso sintético não imunogênico, resposta imune não é solicitada, não necessitando assim fazer teste em pele previamente ao seu uso.

Entretanto, Liu, et al., (2018) relatou um caso clínico de alopécia na paciente que foi submetida a tratamento com CaHA no lado frontal direito do couro cabeludo com o objetivo de elevar a sobrancelha deste lado. Um mês após ter recebido o tratamento, paciente apresentou, uma área de 5 x $4 \mathrm{~cm}$ do lado que recebeu injeção com perda desigual de cabelo. Paciente negou qualquer mudança no seu estilo de vida. Clinicamente a pele estava normal exceto pela atrofia. A histologia encontrada sugeriu corpos de granuloma. Após 6 meses o cabelo voltou a crescer na área com alopécia. Autor conclui sugerindo que CaHA seja injetado no plano da derme ou subderme nestes casos de lift de sobrancelha e não usar mais que $0,3 \mathrm{ml}$ do produto pois quanto mais profundo o nível da injeção no caso do bulbo capilar e quanto maior o volume, maior a possibilidade de formar granuloma e consequentemente alopecia. 


\subsection{Imaginologia}

Koka, et al., (2017) declara que seu artigo foi o primeiro a descrever um preenchedor dérmico radiopaco em paciente que fez tal procedimento com finalidade exclusivamente estética e alerta que com a busca crescente de tratamentos estéticos, os dentistas encontrarão estas imagens cada vez com mais frequência em radiografias intra e extra-orais e em tomografias computadorizadas. Em seu relato, a paciente havia usado Radiesse em setembro de 2015 e uma segunda aplicação em outubro de 2015. Em dezembro de 2015 ela fez uma radiografia panorâmica como exame preliminar para confecção de nova prótese sobre implante, quando foram detectadas as imagens radiopacas. Considerando a experiência do médico da paciente, que observa que $80 \%$ das microesferas de hidroxiapatita de cálcio reabsorvem em 3 a 6 meses e que o restante do material remanescente leva de 8 a 10 meses; se a radiografia tivesse sido feita 10 meses ou mais após o procedimento estético, espera-se que pouco ou nenhuma imagem do material fosse encontrada.

A hidroxiapatita de cálcio também pode ser visualizada em ultrassonografias de alta resolução, segundo Lee (10). Ele relata que este material aparece como depósitos hiperecóicos com níveis variados de sombreamento acústico posterior.

\section{Discussão}

A literatura demonstra que a hidroxiapatita de cálcio tem se apresentado clinicamente bem-sucedida ao longo dos últimos 14 anos. Apesar de a FDA ter aprovado Radiesse® (Merz Aesthetics, Raleigh, NC) em 2006 como um preenchedor á base de hidroxiapatita de cálcio para uso em sulcos naso-labiais e em pacientes com lipoatrofia facial (10), ela também tem sido usada na forma diluída como estimulador de colágeno.

Muitos autores concordam com a eficácia da hidroxiapatita de cálcio como estimulador de colágeno, melhorando as propriedades do tecido. Zerbinati e Calligaro (2018) compararam amostras de tecidos que receberam hidroxiapatita de cálcio com amostras de tecidos, na mesma paciente, que não receberam hidroxiapatita de cálcio. Apesar do " $n$ " ter sido pequeno, pois foram apenas 5 pacientes, eles encontraram uma proporção relativamente maior de colágeno novo (colágeno tipo III) nas amostras de tecido que receberam a hidroxiapatita de cálcio e nas amostras do grupo controle, eles encontraram uma quantidade maior de colágeno maduro, que é o colágeno tipo I, confimando assim que a hidroxiapatita de cálcio estimula a formação de colágeno tipo III. Casabona e Marchese (2017) compararam amostras de tecidos que receberam tratamento com injeções de hidroxiapatita de cálcio diluída 1:1, microagulhamento e ácido ascórbico tópico, com amostras de tecido que receberam apenas microagulhamento e ácido ascórbico tópico, com amostras de tecido que não receberam tratamento algum. $\mathrm{O}$ "n" também é pequeno, pois das 35 pacientes que foram submetidas ao tratamento, apenas 1 seria submetida a uma plástica abdominal e a mesma consentiu com a remoção do tecido para estudo comparativo. Entretanto, as análises histológicas revelaram um aumento na quantidade e na qualidade de colágeno e fibras de elastina nas áreas tratadas com hidroxiapatita de cálcio, microagulhamento e ácido ascórbico em comparação com as amostras que receberam apenas tratamento com microagulhamento e ácido ascórbico e com as amostras de tecido que não receberam tratamento. Também devemos considerar, que das 35 pacientes, 25 tinham estrias vermelhas, ou seja, estrias mais recentes, o que favorece uma resposta positiva ao tratamento. Desta forma, o estudo de Casabona e Marchese (2017) consolida o que Zerbinati e Calligaro (2018) haviam concluído: que o uso da hidroxiapatita de cálcio diluída aumenta a produção de colágeno.

Courderot-Masuyer, et al., (2016) estudaram a força contrátil dos fibroblastos envelhecidos numa pele normal, fibroblastos em sulcos e o efeito da hidroxiapatita de cálcio nestes fibroblastos. Apesar do "n" também ter sido pequeno pois foram 3 pacientes do sexo feminino, entre 49 e 55 anos, que foram submetidas a cirurgia plástica de lift da face e que consentiram com a biopsia de tecido para pesquisa; os autores perceberam um aumento da força contrátil nos fibroblastos que receberam tratamento com hidroxiapatita de cálcio quando comparados com os fibroblastos envelhecidos e com os fibroblastos 
de sulcos. Sendo assim, eles concordam com Casabona e Marchese (2017) quanto a melhora na qualidade dos fibroblastos presentes no tecido quando submetidos ao tratamento com hidroxiapatita de cálcio.

Em 2016, mais de 400 profissionais da área estética, oriundos de 60 países, se reuniram em Praga para um meeting e Fabi, et al., (2017) compilou em sua publicação em 2017 um sumário das apresentações feitas neste encontro. Foram apresentados tratamentos faciais e corporais associando hidroxiapatita de cálcio diluída (Radiesse) com ultrassom microfocado Ulthera e com o sistema Cellfina, este último indicado para tratamento de celulite. O encontro foi promovido pela Merz North America (USA) e os três tratamentos propostos são desta empresa, assim como os autores revelaram relacionamento financeiro com esta e com outras empresas (Allergan, Galderma, Valeant, Palomar, Brickell Biotech, Cutera, Teoxane, Revance, Neothetics, Sienna and Lutronic, Medytox, LG Life Sciences, Daewoong). Entretanto, vale ressaltar que os autores concluíram que a Cellfina para tratamento de celulite e Ultherapy e hidroxiapatita de cálcio diluída para melhora das propriedades mecânicas da pele são tratamentos emergentes nas abordagens inovadoras para rejuvenescimento e embelezamento do corpo e da face. A hidroxiapatita de cálcio diluída mostrou ser um tratamento efetivo para melhora de contorno e firmeza da pele de braços. Ele também mostrou ser efetivo no tratamento de pescoço e colo, melhorando as propriedades mecânicas da pele e estímulo a neocolagenese. O procedimento dispara um processo de neocolagênese, no qual o colágeno tipo I gradualmente substitui o colágeno tipo III, e elastogênese. Corroborando com Zerbinati e Calligaro (2018) que declaram que durante a neocolagênese, o colágeno tipo III recém-formado é substituído gradualmente pelo colágeno maduro tipo I para obter suporte estrutural ideal e resistência à tração.

De Almeida, et al., (2019) chegaram a esta mesma conclusão quando $70 \%$ a $89 \%$ dos 10 especialistas em dermatologia e cirurgia plástica, com grande experiência em preenchedores dérmicos e tratamentos com bioestimuladores para face e corpo, responderam um questionário num Meeting em São Paulo em 2017 e concordaram com o consenso que determina as recomendações de uso da hidroxiapatita de cálcio como bioestimulador para rejuvenescimento de face e corpo, promovendo melhora da qualidade e firmeza da pele.

Em se tratando da hidroxiapatita de cálcio como preenchedor, os autores Lee e Lorenc (2016), Lorenc, et al., (2018) e Goldberg, et al., (2018) avaliaram características físico-químicas do material como módulo de elasticidade, viscosidade e reologia. Todos concordam que o seu módulo de elasticidade é alto, o que significa que a substância tem menor chance de deformar quando submetida à pressão. Deste modo, Lee e Lorenc (2016) ressaltaram que esta propriedade resulta um melhor efeito lift. Lorenc, et al., (2018) concluíram que tanto o módulo de elasticidade quanto a viscosidade da hidroxiapatita de cálcio são maiores em comparação com os preenchedores à base de ácido hialurônico; oferecendo assim, bases científicas para usar a hidroxiapatita de cálcio como um implante líquido e um excelente suporte para as revolumizações da face. Goldberg, et al., (2018) concordaram com Lorenc, et al., (2018) e também sugere o uso de hidroxiapatita de cálcio como suporte para procedimentos complementares como outros preenchedores e uso de fios de sustentação. Eles também concordam que a hidroxiapatita de cálcio pode ser considerada um implante líquido por causa do seu alto módulo de elasticidade, sendo que Goldberg, et al., (2018) inclusive sugere o uso em áreas como mento e região frontal, que são áreas de difícil tratamento.

Quanto à satisfação, Bass, et al., (2009), Casabona e Marchese (2017) e Guida, et al., (2020) concordam que o nível de satisfação dos pacientes é alto; sendo que Bass, et al., (2009) encontraram 94,6\% de satisfação dos pacientes após 6 meses de aplicação de hidroxiapatita de cálcio em sulcos nasolabiais moderados a severos, Casabona e Marchese (2017) observaram $85,8 \%$ de satisfação nos pacientes que usaram hidroxiapatita de cálcio associado a microagulhamento e ácido ascórbico $20 \%$ em estrias vermelhas e brancas e Guida, et al., (2020) encontraram 100\% de satisfação das pacientes que receberam CaHA diluida 1:4 na região acima dos joelhos para tratamento de flacidez e celulite. Enquanto Liu, et al., (2018) avaliando uso da hidroxiapatita de cálcio para lift de sobrancelha, observou que a paciente não percebeu nenhum resultado com o tratamento realizado, e além disso teve alopecia na região do couro cabeludo onde foi aplicado o produto, mas após 6 meses, o cabelo 
voltou a crescer. Eles concluíram que nas regiões de bulbo capilar, existe uma chance maior de formar granuloma com consequente alopecia quanto mais profundo for o plano de aplicação e quanto maior volume for aplicado. Recomendando assim, aplicar na derme ou subderme e não mais que $0,3 \mathrm{ml}$.

Em se tratando de segurança, a terapia com hidroxiapatita de cálcio é considerada segura de acordo com os autores e fazem algumas considerações como evitar o uso em áreas dinâmicas como região orbicular dos lábios e olhos, assim como glabela e nariz que são áreas de maior risco onde devemos ter cautela em qualquer tipo de procedimento. Da mesma forma, Loghem, et al., (2015) concordam que a hidroxiapatita de calcio tem indicação de uso para todas as áreas da face, exceto, glabela, região periorbital e lábios.

Como efeito adverso, os mais comuns são eritema, edema e equimose que desaparecem em poucos dias, e Kaudoch, (2017), De Almeida, et al., (2019) e Lee e Lorenc (2016) incluem os nódulos, mas que em sua grande maioria são resolvidos com massagem manual e corticoide intralesional. Apenas Kaudoch (2017) relata que em dez nódulos que foi considerada necessária a excisão dos mesmos, mas não foi relatado quantos nódulos foram efetivamente removidos. De Almeida, et al., (2019) e Kaudoch (2017) relatam que necrose tecidual e embolismo não foram observados na prática clínica; e Lee e Lorenc (2016) considera oclusão vascular extremamente rara e quando acontece, sugere usar o mesmo protocolo utilizado para outros preenchedores, incluindo o uso de hialuronidase. Lee e Lorenc (2016) também consideram desnecessário teste de pele prévio ao uso da hidroxiapatita de cálcio, pois sendo considerado um osso sintético não imunogênico, não há resposta imune do organismo com a sua presença.

Com o crescente aumento do uso da hidroxiapatita de cálcio, é importante a busca de um agente de reversão para ele, assim como existe a hialuronidase para o ácido hialurônico. Alguns autores estudam um agente de reversão para os casos de redução de nódulo, desvio de material, redução de volume de sobrecorreção e oclusão vascular.. Rullan, et al., (2019) relataram dois casos clínicos de nódulos visíveis de hidroxiapatita de cálcio, cujo tratamento com o tiossulfeto de sódio foi bemsucedido. Entretanto, Danysz, et al., (2020) em seu estudo pré-clinico, concluíram que apesar do tiossulfeto de sódio ter apresentado uma eficácia maior que a solução salina, o uso da solução salina ainda é mais seguro pois o uso do tiossulfeto de sódio apresentou risco de necrose tecidual e hemorragia. Além disso, o mecanismo de ação do tiossulfeto de sódio sugere ser de dispersão e não de degradação, desta forma não ajudaria nos casos de oclusão vascular.

As microesferas de hidroxiapatita são uma forma sintética de uma substância que encontramos naturalmente em ossos e dentes e portanto, são radiopacas quando vistas em radiografias e tomografias. Tendo em vista o crescente número de pacientes que têm recorrido a terapias de rejuvenescimento, o cirurgião dentista deve fazer este diagnóstico diferencial ao avaliar as radiografias intra e extra-orais e também as tomografias, lembrando que estas imagens estarão presentes enquanto o material ainda não tiver sido reabsorvido pelo organismo. Lee e Lorenc (2016) relatou que em exames de ultrassonografia de alta resolução, esta substância também pode ser identificada. Neste caso, a hidroxiapatita aparece como depósitos hiperecóicos com níveis variados de sombreamento acústico posterior.

\section{Conclusão}

Diante do exposto, destaca-se que:

- A hidroxiapatita de cálcio diluída é eficaz como bioestimulador de colágeno e elastina, pois apresentou bases científicas de melhora das propriedades mecânicas da pele, sendo eficaz para aumento da qualidade e firmeza da mesma.

- Como material preenchedor, a hidroxiapatita de cálcio apresentou um melhor efeito lift (exceto para sobrancelha) por causa de seu alto módulo de elasticidade e sugere seu uso como suporte para procedimentos como outros 
preenchedores e fios de sustentação, podendo ser usada como implante líquido em áreas de difícil tratamento como mento e região frontal.

- O uso da hidroxiapatita de cálcio e do ácido hialuronico na mesma sessão apresentou um nível de satisfação maior do que quando o ácido hialuronico é usado separadamente, entretanto precisamos de mais estudos das propriedades reológicas resultantes destes materiais misturados previamente à injeção.

- O nível de satisfação dos pacientes, com o uso da hidroxiapatita de cálcio foi alto, exceto no caso de lift de sobrancelha.

- É considerado um material com um bom perfil de segurança, desde que respeitada a técnica e as áreas que devem ser usadas, evitando áreas dinâmicas como orbicular dos lábios e olhos, e regiões de glabela e nariz que requer cautela em qualquer tipo de procedimento.

- Apesar do tiossulfeto de sódio estar sendo sugerido para o tratamento de redução de volume de sobrecorreção, desvio de material, redução de nódulo e oclusão vascular; maiores estudos se fazem necessários para termos um bom perfil de segurança além de eficácia.

- Sendo um material radiopaco, aparecerá em radiografias e tomografias enquanto não for reabsorvido pelo organismo e o cirurgião-dentista deve fazer o diagnóstico diferencial. Ele também é visualizado em ultrassonografias de alta resolução como depósitos hiperecóicos, com níveis variados de sombreamento acústico posterior.

\section{Referências}

Bass, L. S., et al (2009). Calcium Hydroxylapatite (Radiesse) for Treatment of Nasolabial Folds: Long-Term Safety and Efficacy Results. Aesthetic Surg J. 30(2).235-238.

Casabona, G., \& Marchese P (2007). Calcium Hydroxylapatite Combined with Microneedling and Ascorbic Acid is Effective for Treating Stretch Marks. PRS Global Open. 5(9).1-9.

Courderot-Masuyer, C., et al (2016). Evaluation of lifting and antiwrinkle effects of calcium hydroylapatite filler. In vitro quantification of contractile forces of human wrinkle and normal aged fibroblastos treated with calcium hydroxylapatite. J Cosmetic Dermatol.15(3). $260-268$.

Danysz, W., Nowag, B., Hengl, T., Kreymerman, P., Furne, C., Madeuf, E., \& Robinson, D. M. (2020). Can sodium thiosulfate act as a reversal agent for calcium hydroxylapatite filler? Results of a preclinical study. Clinical, Cosmetic and Investigational Dermatology. $13,1059$.

De Almeida, A. T., et al (2019). Consensus Recommendations for the Use of Hyperdiluted Calcium Hydroxyapatite (Radiesse) as a Face and Body Biostimulatory Agent. PRS Global Open.7(3).1-9.

Fabi, S., et al (2017). Combined aesthetic interventions for prevention of facial ageing, and restoration and beutification of face and body. Clin Cosmetic Inves Dermatol.10:423-429.

Fakih-Gomez, N., \& Kadouch, J. (2021). Combining calcium hydroxylapatite and hyaluronic acid fillers for aesthetic indications: efficacy of an innovative hybrid filler. Aesthetic plastic surgery. 1-9.

Goldberg, D. J. et al (2018). Expanding Treatment Options for Injectable Agents. Aesthetic Surg J. 38(S1): S1-S7.

Guida, S., Longhitano, S., Shaniko, K., Galadari, H., Chester, J., Ciardo, S., \& Farnetani, F. (2020). Hyperdiluted calcium hydroxylapatite for skin laxity and cellulite of the skin above the knee: A pilot study. Dermatologic Therapy. 33(6).e14076.

Guida, S., Longhitano, S., Spadafora, M., Lazzarotto, A., Farnetani, F., Zerbinati, N., \& Galadari, H. (2021). Hyperdiluted calcium hydroxylapatite for the treatment of skin laxity of the neck. Dermatologic Therap. e15090.

Kadouch, J. A., (2017). Calcium hydroxylapatite: A review on safety and complications. J Cosmetic Dermatol.16(2).152-161.

Koka, S., et al (2017). Derma Filler Presenting as Lobular Radiopacities in na Edentulous Patient: A Clinical Report. J Prosthodontics.26(8).670-671.

Lee, J. C., \& Lorenc, Z. P (2016). Synthetic Fillers for Facial Rejuvenation. Clin Plastic Surg.43(3).497-503.

Liu, R., et al (2018). Alopecia with foreign body granulomas induced by Radiesse injection: A case report. J Cosmetic Laser Therapy. 20 (7-8).462-464

Lorenc, Z. P., et al (2018). Physiochemical Characteristics of Calcium Hydroxylapatite (CaHA).201 Aesthetic Surg J. 38(S1). S8-S12. 
Research, Society and Development, v. 10, n. 14, e05101421689, 2021

(CC BY 4.0) | ISSN 2525-3409 | DOI: http://dx.doi.org/10.33448/rsd-v10i14.21689

Rullan, P. P., Olson, R., \& Lee, K. C. (2020). The use of intralesional sodium thiosulfate to dissolve facial nodules from calcium hydroxylapatite. Dermatologic Surgery. 46(10).1366-1368.

van Loghem, J., Funt, D., Pavicic, T., Goldie, K., Yutskovskaya, Y., Fabi, S., \& Saeed, P. (2020). Managing intravascular complications following treatment with calcium hydroxylapatite: an expert consensus. Journal of cosmetic dermatology. 19(11). 2845-2858.

Van Loghem, J., Yutskovskaya, Y. A., \& Werschler, W. P. (2015). Calcium hydroxylapatite: over a decade of clinical experience. The Journal of clinical and aesthetic dermatology. 8(1). 38.

Wollina, U., \& Goldman, A. (2020). Long lasting facial rejuvenation by repeated placement of calcium hydroxylapatite in elderly women. Dermatologic Therapy. 33(6). e14183.

Zerbinati, N. \& Calligaro, A., (2018). Calcium hydroxylapatite treatment of human skin: evidence of collagen turnover through picrosirius red staining and circulary polarized microscopy. Clin Cosmetic Inves Dermatol.11.29-35. 